Reprod. Nutr. Dévelop., 1981, 21 (3), 383-390.

\title{
Purification par chromatographie liquide de haute pression (HPLC) de l'hormone cérébrale chez Nereis diversicolor et Perinereis cultrifera (Annélides Polychètes)
}

par C. CARDON, M. DURCHON, M. PORCHET

Laboratoire de Biologie Animale, Laboratoire Associé au CNRS no 148 ef Laboratoire de Chimie Biologique III, Université des Sciences et Techniques de Lille 59655 Villeneuve d'Ascq Cedex, France.

Summary. Purification of the brain hormone of Nereis diversicolor and Perinereis cultrifera (Annelida : Polychaeta) by high-pressure liquid chromatography (HPLC).

Five thousand brains inhibiting gametogenesis in Nereis diversicolor and Perinereis cultrifera were extracted with cold methanol, giving a micromolecular fraction containing the whole of the inhibitory activity of the brains. This micromolecular fraction, fractionated (i) on Sephadex G 25 fine and (ii) on Sephadex G 25 superfine, gave an inhibitory peak (P 5) in organ culture.

Fraction P 5, purified by high-pressure liquid chromatography, showed an area with the same biological inhibitory activity as the blank brains.

\section{Introduction.}

Chez les Néréidiens, le cerveau contrôle le développement somatique ainsi que la gamétogenèse, par l'intermédiaire d'une hormone libérée au niveau des cellules neurosécrétrices (Durchon, 1948, 1967 ; Hauenschild, 1956, 1965 ; Clark et Ruston, 1963 ; Schröder, 1966 ; Dhainaut-Courtois, 1968).

Au moment de la maturité génitale l'animal subit une véritable métamorphose somatique : l'épitoquie. La Nereis porte alors le nom d'Heteronereis.

Pour les espèces présentant naturellement une épitoquie, cette hormone inhibe chez les deux sexes les transformations morphologiques et anatomiques et empêche les animaux de devenir Heteronereis; une décérébration provoque une épitoquie prématurée Perinereis cultrifera (Durchon, 1948, 1952, 1956) ; Nereis irrorata (Durchon, 1952) ; Perinereis marionii (Durchon, 1952) ; Nereis pelagica (Choquet, 1962) ; Platynereis dumerilii (Hauenschild, 1964a) et Nereis succinea (Malecha, 1967).

Chez toutes les espèces cet effet inhibiteur s'exerce, aussi, sur la gamétogenèse des deux sexes. Chez les mâles, l'ablation du cerveau déclenche la spermatogenèse ; 
elle aboutit à la libération des spermatozoïdes (Durchon, 1962). Chez les femelles, par contre, l'évolution des cellules sexuelles en l'absence d'hormone inhibitrice est plus nuancée et varie selon les espèces (Hauenschild, 1956 ; Clark et Ruston, 1963 ; Choquet, 1962).

L'activité hormonale du cerveau diminue régulièrement au cours de la vie de l'annélide, pour finalement devenir nulle à l'approche de la reproduction (Durchon, 1960, 1962 ; Havenschild, 1964a, b; Durchon et Porchet, 1971).

A partir de ces résultats notre travail a été consacré à l'isolement et à la purification de l'hormone. A ce jour, aucun auteur n'a pu en effet obtenir la preuve expérimentale de l'existence de plusieurs hormones au niveau du cerveau (Durchon ef Joly, 1978). Le problème de l'unicité ou non du principe hormonal ne doit pas cependant être considéré comme résolu. Dans ce but nous exposerons une méthode de purification par chromatographie liquide de haute pression (HPLC).

\section{Matériel et méthodes.}

Il est indispensable pour extraire et caractériser une substance hormonale de disposer d'un test biologique dont les données soient répétitives. Ce test est basé sur l'évolution in vitro des spermalogonies en absence ou en présence d'hormone cérébrale (Durchon et Schaller, 1964). Le principe de cette méthode consiste à mettre en culture organotypique des parapodes de Nereis diversicolor, contenant des spermatogonies, en absence (cultures isolées de parapodes) ou en présence d'hormone (association parapode-cerveau).

En culture isolée, donc en milieu anhormonal (température : $20^{\circ} \mathrm{C}$ ), les spermatogonies subissent la méiose au $3^{\mathrm{e}}$ jour. Le stade de spermatozoïde est atteint au $6 \mathrm{e}$ jour. Dans le cas d'une association parapode-cerveau, donc en présence d'hormone, cette dernière assure un blocage de la méiose ; un mois plus tard, les spermatogonies n'ont subi aucune évolution.

Dans ces conditions, pour évaluer l'efficacité des fractions isolées par voie biochimique, des cultures organotypiques sont effectuées $\left(a ̀ 0^{\circ} \mathrm{C}\right.$ ) : d'une part, des cultures témoins, parapodes isolés dans lesquels les spermatogonies subissent leur passage au stade de spermatozoïdes en 6 jours. Une deuxième catégorie de témoins est constituée pat des associations cerveau-parapode, pour lesquelles la spermatogenèse doit être absente après un mois de culture pour que l'ensemble de l'expérimentation soit pris en considération (Durchon et Schaller, 1964).

Enfin, les autres séries expérimentales de cultures sont constituées de parapodes, prélevés sur le même animal, ef placés sur un milieu de culture contenant une fraction biochimique donnée. Dans le cas où cette dernière est efficace, on enregistre un retard dans le temps mis par les spermatogonies pour accéder à la spermatogenèse ( $X$ jours). Le degré d'efficacité des différentes fractions est évalué en fonction du temps $X+6$ jours (par rapport aux témoins); plus la valeur de $X$ est grande, plus l'efficacité de la fraction est élevée.

In vivo, l'ablation des ganglions cérébroïdes déclenche la maturation génitale chez les deux sexes; en outre, tous les caractères somatiques de l'épitoquie naturelle apparaissent chez des individus jeunes ayant subi cette opération. Une injection intra- 
cœlomique de la fraction hormonale suffit à prévenir maturation génitale ef épitoquie prématurées.

Préparation du matériel brut. -5000 cerveaux prélevés sur des individus jeunes, sont homogénéisés dans $20 \mathrm{ml}$ d'eau bidistillée. A l'homogénat sont ajoutés $300 \mathrm{ml}$ de méthanol redistillé refroidi à $-70^{\circ} \mathrm{C}$; on obtient une précipitation des macromolécules. Après centrifugation de l'homogénat à $3000 \mathrm{~g}$ pendant $10 \mathrm{~min}$, on obtient un sédiment et un surnageant. L'opération est répétée 3 fois sur le sédiment. On récupère, à chaque fois, le surnageant.

En fin d'expérience on a : une fraction insoluble et une fraction méthanolique constituée par l'ensemble des surnageants. Le méthanol est éliminé à l'évaporateur rotatif. La fraction aqueuse résiduelle est alors délipidée par l'éther dépourvu de péroxyde.

On obtient finalement deux phases : $1^{\circ}$ une phase éthérée, $2^{\circ}$ une phase aqueuse. La phase éthérée nous fournit la fraction lipidique et la phase aqueuse la fraction micromoléculaire (Durchon, Montreuil et Boilly-Marer, 1963) responsable de l'activité biologique. On entend par fraction micromoléculaire, l'ensemble des constituants qui n'ont pas précipité sous l'action du méthanol et qui restent solubles dans l'eau.

Fractionnement du facteur micromoléculaire hormonal par tamisage moléculaire. Une quantité de fraction « micromoléculaire » correspondant à 1500 cerveaux est dissoute dans $1 \mathrm{ml}$ d'eau bidistillée. Cette solution est chromatographiée sur une colonne de gel de sephadex (hauteur $114 \mathrm{~cm}$; diamètre $1 \mathrm{~cm}$ ) G 25 « normal » ou « medium » et G 25 " super-fine » (Cardon, 1970). Le déplacement des composés est effectué par passage d'eau bidistillée.

Après chromatographie sur G 25 normal une fraction $P 2$ possède toute l'activité biologique.

Cette fraction P 2 esi alors purifiée sur G 25 « super-fine »; seuls les pics P 5 ef $P 6$ se montrent alors inhibiteurs de la spermatogenèse.

Détermination de la masse moléculaire de la fraction hormonale par tamisage moléculaire. - On a utilisé une colonne de Sephadex « G 25 fine » $(1,3 \times 100 \mathrm{~cm}$ ) (Andrews, 1964), équilibrée par une solution de tampon acétate $0,05 \mathrm{M}$ additionnée de $0,4 \mathrm{M}$ de $\mathrm{NaCl}$ à $20^{\circ} \mathrm{C}$ (Kanatani et al., 1971).

Le volume d'élution de la fraction active est comparé d'une part à celui de la bacitracine par détermination des courbes d'absorption en lumière ultraviolette à $254 \mathrm{~nm}$ et $280 \mathrm{~nm}$, d'autre part à celui de l'ocytocine. Dans ce dernier cas on a recours à l'essai biologique sur un utérus isolé de rate en prostrus. La masse moléculaire estimée par ce procédé est de l'ordre de 2000 daltons.

Purification par chromatographie de haute pression. - L'appareil est un chromatographe « Waters associate » M $6000 \mathrm{~A}$, muni d'un injecteur à boucle de type $U 6 \mathrm{~K}$, d'un détecteur réfractométrique « Differentiel refractometer $R 401$ » et d'un détecteur photométrique à $254 \mathrm{~nm}$ (model 440 absorbance detector). L'enregistrement sur papier est réalisé à l'aide d'un «Omniscribe recorder Houston instrument».

La colonne chromatographique est une $\mu$-bondapak $C 18$ Waters assoc. dont les dimensions sont les suivantes : diamètre inférieur $3,9 \mathrm{~mm}$; longueur $30 \mathrm{~cm}$. 
La phase mobile consiste en une solution à 40 p. 100 de méthanol distillé, dégazé dans l'eau bidistillée, elle-même dégazée. Le débit est de $1,4 \mathrm{ml} / \mathrm{min}$. La pression en tête de colonne est d'environ 1100 p.s.i. La chromatographie est réalisée à la température du laboratoire (de l'ordre de $20^{\circ} \mathrm{C}$ ).

La fraction $P 5$, résultat de la chromatographie d'une fraction micromoléculaire correspondant à 1500 cerveaux sur « $G 25$ » normal et $G 25$ super-fine, est reprise par $5 \mathrm{ml}$ d'eau bidistillée ; cette solution est centrifugée à $5000 \mathrm{tr} / \mathrm{min}$ pendant $5 \mathrm{~min}$. On récupère le surnageant, le culot est éliminé. Le surnageant est lyophilisé, repris par $0,6 \mathrm{ml}$ d'eau bidistillée ; il est filtré sur millipore $0,45 \mu$, on ajoute au filtrat $0,4 \mathrm{ml}$ de méthanol redistillé. On obtient ainsi un extrait de 1500 cerveaux dans $1 \mathrm{ml}$ de phase mobile.

A l'aide d'une seringue Hamilton spéciale haute pression, on réalise une suite d'injections de $25 \mu$ l, ce qui correspond à un extrait équivalent de 37 cerveaux. Les fractions correspondantes sont rassemblées et après élimination du méthanol à l'évaporateur rotatif, elles sont lyophilisées, ef testées en culture organotypique.

\section{Résultats.}

Les trois fractions : insoluble, éthérée et aqueuse ont été étudiés in vitro et in vivo. In vitro le test utilisé est l'évolution des spermatogonies contenues dans les parapodes explantés sur des Nereis diversicolor mâles immatures et cultivés isolément (Durchon et Schaller, 1963, 1964). Lorsque la fraction micromoléculaire (équivalent de 15 cerveaux) est incorporée au milieu de culture, la spermatogenèse est retardée de 11 jours par rapport aux témoins. Les autres fractions sont inactives (Cardon, 1970). In vivo, des animaux décérébrés (Perinereis cultrifera) reçoivent des injections de ces différentes fractions. Seuls les individus ayant reçu des injections de la fraction micromoléculaire ne présentent ni maturation génitale ni épitoquie précoces.

D'après ces résultats, on peut considérer que l'hormone inhibitrice est présente dans la fraction micromoléculaire.

\section{Fractionnement par tamisage moléculaire.}

Les constituants de la fraction « micromoléculaire » sont séparés par chromatographie sur colonne en utilisant des supports tels que le Sephadex G 25 «fine » ou " medium ». Cette séparation par tamisage moléculaire fournit un diagramme sur lequel se dessinent de nombreux pics (fig. 1). Cette chromatographie est reproductible.

Les différentes fractions qui absorbent la lumière ultraviolette ef qui correspondeni aux pics, sont isolées et incorporées au milieu de culture. Seule la fraction P 2 possède un pouvoir inhibiteur. Elle inhibe la spermatogenèse pendant 6 à 7 jours (pour une concentration équivalente à 15 cerveaux).

La fraction $P 2$, est sous-fractionnée sur $G 25$ « super-fine ». Le diagramme obtenu comporte de nombreux pics (fig. 2). Seuls les deux derniers, parfaitement séparés se sont montrés inhibiteurs de la spermatogenèse : ce sont les pics 5 et 6 .

La fraction P 5 a un effet inhibiteur pendant 6 à 7 jours ; la fraction P 6 pendant 5 à 6 jours, soit $24 \mathrm{~h}$ de moins que la précédente. 


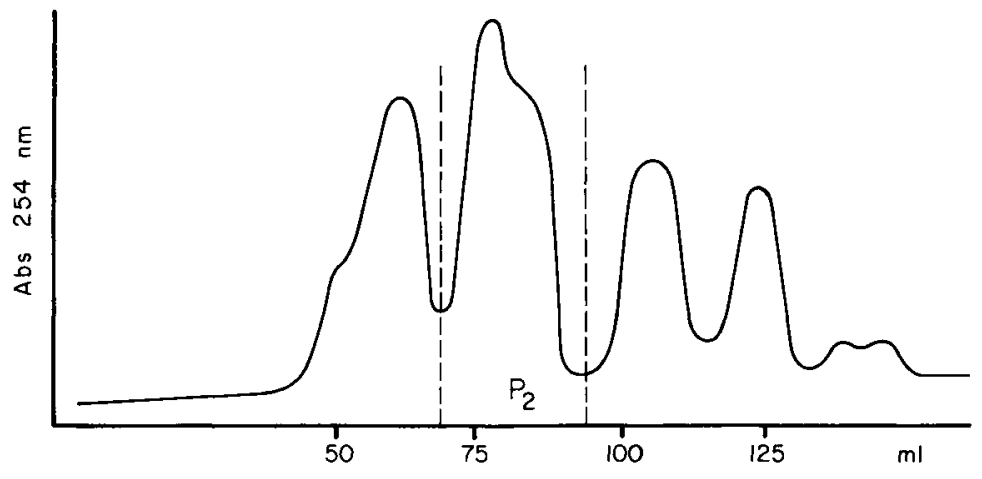

FIG. 1. - Fractionnement de la fraction « micromoléculaire » sur gel de Sephadex $G 25$ « medium ». Colonne $(114 \mathrm{~cm} \times 1) ; 15 \mathrm{mg}$ de fraction micromoléculaire dans $1 \mathrm{ml}$ d'eau. Eluant : eau bidistillée ; vitesse d'élution $8 \mathrm{ml} / \mathrm{h}$; absorbance mesurée en continu à $254 \mathrm{~nm}$.

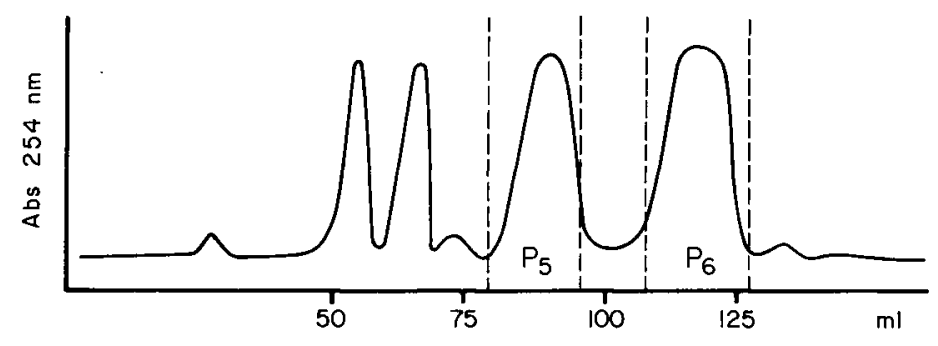

FIG. 2. - Fractionnement de $P 2$ sur gel de Sephadex G 25 « super fine ».

Colonne $(114 \mathrm{~cm} \times 1) ; 15 \mathrm{mg}$ de fraction $P 2$ dans $1 \mathrm{ml}$ d'eau. Eluant : eau bidistillée ; vitesse d'élution $8 \mathrm{ml} / \mathrm{h}$; absorbance mesurée en continu à $254 \mathrm{~nm}$.

FIG. 3. - Isolement d'une fraction intermédiaire entre $P 5$ et $P 6$.

Des mises en cultures ont été pratiquées avec des fractions correspondant au maximum d'absorption des fractions $P 5$ et $P$ et avec la fraction intermédiaire c'est-àdire la zone qui correspond au retour à la ligne de base (fig. 3). Nous avons alors constaté un maximum de retard pour la fraction intermédiaire et $P 5$. Celles-ci inhibent, en effet, la spermatogenèse deux fois plus longtemps que la fraction $\mathbf{P} 6$.

Les retards enregistrés, lors des précédentes mises en cultures avec les fractions correspondant à $P 5$ et $P 6$ s'expliquent parfaitement. Au moment de la séparation en fractions 5 et 6 , chacune d'elles contenait une quantité plus ou moins grande de produit actif.

En effectuant un isolement des fractions tube par tube et en incorporant chacune d'elles au milieu de culture, nous avons obtenu par enregistrement spectrophotométrique des courbes, qui, superposées, donnent une courbe d'activité biologique qui délimite exactement la fraction hormonale (fig. 4). 


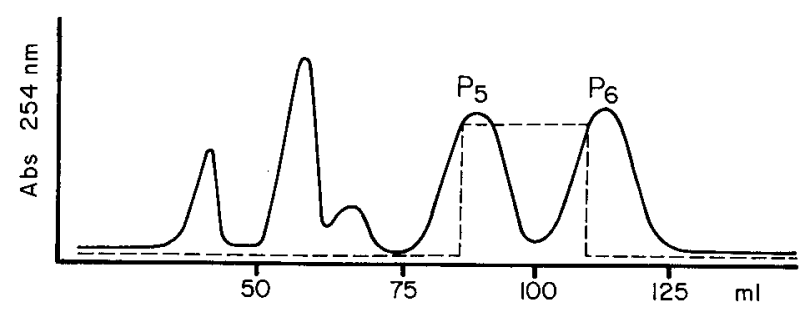

FIG. 4. - Fractionnement de P 2 sur « Sephadex G 25 super-fine ».

En trait continu : absorbance mesurée en continu à $254 \mathrm{~nm}$; tracé discontinu : activité biologique.

Purification par chromatographie liquide-liquide de haute pression.

La fraction hormonale se situe au niveau des pics $P 5$ et $P 6$. Cette répartition se fait à 70 p. 100 dans le pic 5 et la zone intermédiaire 5-6 (fig. 4).

Lorsque $P 5$, zone intermédiaire, et $P 6$ sont rassemblés et incorporés aux cultures organotypiques la spermatogenèse reste bloquée 10 à 11 jours. Par contre si on ne conserve que la fraction correspondant à $P$ et à la zone intermédiaire, la spermatogenèse reste bloquée 6 à 7 jours. On en déduit donc que 70 p. 100 de l'activité biologique est rassemblée au niveau du pic 5 et de la zone intermédiaire.

Nous avons essayé d'améliorer le procédé de purification de cette fraction hormonale par chromatographie liquide de haute pression.

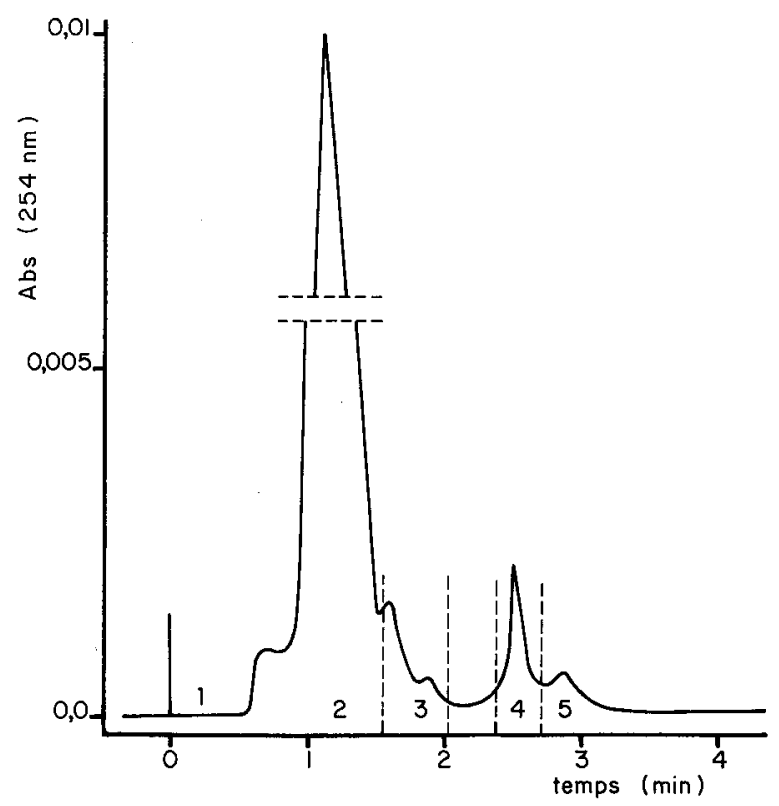

FIG. 5. - Résultaf d'analyse de la fraction P 5 sur colonne $\mu$-bondapak $C$ 18. La phase mobile consiste en méthanol-eau $(40: 60 \mathrm{~V} / \mathrm{V})$. Le débit est de $1,4 \mathrm{ml} / \mathrm{min}$. La pression en tête de colonne est de 1100 p.s.i. L'absorbance est mesurée à $254 \mathrm{~nm}$. Volume injecté : $25 \mu \mathrm{l}$. Vitesse de déroulement du papier : $5 \mathrm{~cm} / \mathrm{min}$. 
Les neurohormones étant de nature peptidique, notre choix s'est porté sur une colonne $\mu$-bondapak-C 18 susceptible de séparer des peptides.

Quatre phases mobiles ont été essayées :

- méthanol-eau-acide acétique 50:50:1, V/V ;

- méthanol-eau-acide orthophosphorique 50:50:0,1, V/V ;

— acétonitrile-eau-acide orthophosphorique 25:75:0,1, V/V ;

- méthanol-eau 40:60, V/V.

(Hancock et al., 1978 ; Hearn, Hancock et Bishop, 1978.)

La chromatographie liquide-liquide de haute pression, à l'aide de cette dernière phase mobile, nous a donné pour chaque injection 5 fractions majeures et des diagrammes particulièrement reproductibles (fig. 5). L'incorporation de chacune d'elles dans les milieux de culture nous a permis de déterminer la zone d'activité biologique. II semble que l'activité maximum soit rassemblée au niveau du pic 4 (6 jours d'inhibition de la gamétogenèse) et décroît au niveau du pic 5 (4 jours d'inhibition).

\section{Conclusions.}

L'isolement de la neurohormone d'origine cérébrale des Néréidiens a été réalisé à partir de cerveaux prélevés sur des Nereis diversicolor immatures.

Nos résultats permettent d'avancer les conclusions suivantes:

L'extraction méthanolique des cerveaux fournit 3 fractions (macromoléculaire, lipidique et micromoléculaire); seule la fraction micromoléculaire possède une activité biologique in vivo et in vitro. Cet extrait micromoléculaire est fractionné par tamisage moléculaire. Ce procédé de fractionnement et de sous-fractionnement par filtration sur gel permet d'obtenir deux fractions (5 et 6 ) actives. Une mise en culture tube par tube nous a révélé que le principe actif était réparti à 70 p. 100 au niveau du pic 5 et entre les pics 5 et 6 . La méthode de fractionnement sur gel de sephadex nous a permis d'estimer la masse moléculaire à 2000 daltons.

Cette fraction active est purifiée de façon très satisfaisante par chromatographie préparative de haufe pression. On obtient, par cette méthode, 5 pics, dont l'un (le pic 4) possède la majorité de l'activité biologique. II faut souligner, que cette fraction correspond à moins de 5 cerveaux ef qu'elle permet d'inhiber la spermatogenèse pendant 6 jours, in vitro. Actuellement, le problème de l'isolement de l'hormone inhibitrice cérébrale des Néréidiens semble être en partie résolu ; par contre celui de sa nature chimique demeure posé. Nous espérons que la fraction 4 issue de la chromatographie en HPLC est dans un état de pureté suffisant, pour envisager l'application de méthodes analytiques moléculaires (spectrophotométrie de masse, spectrophotométrie en infra-rouge, utilisation possible de la RMN) et accéder à la connaissance de sa nature et de sa structure chimique.

Reçu en juillet 1980.

Accepté en novembre 1980.

\section{Références}

ANDREWS P., 1964. Estimation of the molecular weights of proteins by Sephodex Gel-filtration. Biochem. J., 91, 222-233. 
CARDON C., 1970. Procédés de fractionnement de ganglions cérébroïdes de Nereis diversicolor O. F. Müller en vue de l'isolement de l'hormone inhibitrice de la sexualisation. Bull. Soc. Zool. Fr., 95, 543-549.

CHOQUET M., 1962. Effet inhibiteur de l'hormone cérébrale sur l'évolution des cellules sexuelles chez Nereis pelagica L. (Annélide Polychète). C. R. Soc. Biol., 156, 1112-1114.

CLARK R. B., RUSTON R. J. G., 1963. The influence of brain extirpation on oogenesis in the polychaete Nereis diversicolor. Gen. comp. Endocr., 5, 529.541.

DHAINAUT-COURTOIS N., 1968. Ełude histologique ef ultrastructurale des cellules nerveuses du ganglion cérébral de Nereis pelagica L. (Annélide Polychète). Comparaison entre les types celIulaires I-VI et ceux décrits antérieurement chez les Nereidae. Gen. comp. Endocr., 11, 414-443.

DURCHON M., 1948. Epitoquie expérimentale chez deux polychètes : Perinereis cultrifera ef Nereis irrorata Malmgren. C. R. Acad. Sci. Paris, 227, 157-158.

DURCHON M., 1952. Recherches expérimentales sur deux aspects de la reproduction chez les Annélides Polychètes : l'épitoquie et la stolonisation. Ann. Sci. nat., 11e sér., 14, 119-206.

DURCHON M., 1956. Rôle du cerveau dans la maturation génitale et le déclenchement de l'épitoquie chez les Néréidiens. Ann. Sci. nat. Zool. Biol. anim., 18, 269-273.

DURCHON M., 1960. L'endocrinologie chez les Annélides Polychètes. Bull. Soc. Zool. Fr., 85, $275-301$.

DURCHON M., 1962. Induction et inhibition expérimentale de l'épitoquie par homogreffes chez les Néréidiens. Bull. Soc. Zool. Fr., 87, 575-582.

DURCHON M., 1967. L'endocrinologie des vers ef des mollusques. Masson ef Cie éd., Paris.

DURCHON M., JOLY P., 1978. L'endocrinologie des Invertébrés. P.U.F., éd. Paris, pp. 235.

DURCHON M., MONTREUIL J., BOILLY-MARER Y., 1963. Résulfats préliminaires sur la nature chimique de l'hormone inhibitrice du cerveau des Néréidiens (Annélides Polychètes). $C$. R. Acod. Sci. Paris, sér. D, 257, 1807-1808.

DURCHON M., PORCHET M., 1971. Premières données quanfitatives sur l'activité endocrine du cerveau des Néréidiens au cours de leur cycle sexuel. Gen. comp. Endocr., 16, 555-565.

DURCHON M., SCHALLER F., 1963. Application de la méthode de culture organotypique aux recherches endocrinologiques chez les Annélides polychètes. C. R. Acad. Sci. Poris, 256, 5615-5617.

DURCHON M., SCHALLER F., 1964. Recherches endocrinologiques en culture organotypique chez les Annélides Polychètes. Gen. Comp. Endocr., 4, 427-432.

HANCOCK W. S., BISHOP C. A., PRESTIDGE R. L., HARDING D. R. K., HEARN M. T. W., 1978. Reversed-phase high-pressure liquid chromatography of peptides and proteins with ionpairing reagents. Sciences, 200, 1168-1170.

HAUENSCHILD C., 1956. Hormonal Hemmung der Geschlechtsreife und Metamorphose bei dem Polychaefen PI. dumerilii. Z. Naturforsch., 11b, 125-132.

HAUENSCHILD C., 1964a. Postembryonale Entwicklungssteuerung durch ein Gehirn-hormon bei PI. dumerilii. Zool. Anz., suppl. 27, 111-120.

HAUENSCHILD C., 1964b. L'influence de l'hormone du cerveau sur le développement postembryonnaire de Platynereis dumerilii. Ann. Endocr., 25, 49-56.

HAUENSCHILD C., 1965. Hormon bei Nereiden und anderen niederen Wirbellosen. Zool. Jb. Abt. allg. Zoo. Physiol. Tiere, Sisch., 71, 511-544.

HEARN M. T. W., HANCOCK W. S., BISHOP C. A., 1978. High-Pressure liquid chromatographyamino-acids, peptides and proteins. $V$. Separation of thyroidal iodo-amino acids by hydrophilic ion-paired reversed-phase high-performance liquid chromatography. J. Chromatogr., 157, 337-344.

KANATANI H., IKEGAMI S., SHIRAL H., OIDE H., TAMURA S., 1971. Purification of gonad stimulating substance obtained from radial nerves of the starfish Asterias amurensis. Dev. Growth Differ., 13, $151-164$.

MALECHA J., 1967. Transformation hétéronéréidienne et gamétogenèse chez Nereis succinea Leuckart (Annélide Polychète) en culture organotypique. C. R. Acad. Sci. Paris, sér. D, 265, 613-615.

SCHRÖDER P. C., 1966. A histological and autoradiographic study of normal and induced metamorphosis in the nereid polychaete Nereis grubei (Kinberg). Doct. diss., Stanford Univ. 